\title{
Optical coherence tomography in forensic sciences: a review of the literature
}

\author{
Matteo Nioi ${ }^{1}$ (D) $\cdot$ Pietro Emanuele Napoli ${ }^{2} \cdot$ Sarah Michelle Mayerson ${ }^{1} \cdot$ Maurizio Fossarello $^{2} \cdot$ Ernesto d'Aloja $^{1}$
}

Accepted: 7 June 2019 / Published online: 18 July 2019

(C) Springer Science+Business Media, LLC, part of Springer Nature 2019

\begin{abstract}
Optical coherence tomography (OCT) is an interferometric imaging technique that has revolutionized clinical ophthalmology since the first half of the 1990's. Despite this approach being successfully employed in ophthalmology and having great potential in forensic cases, its use in different forensic fields appears to be quite limited. In this review we reviewed the scientific literature regarding the application of OCT in forensic science and legal medicine from 1995 to 2019. Our research showed the usefulness of this approach for the study of coronary injuries, postmortem ocular changes, forensic entomology, and several other applications of specific forensic interest (the study of blood stains, fingerprints, and hair bulbs for personal identification, as well as the study of materials found in the crime scene for comparation, or anti-fraud investigation). The creation of specific 'ad hoc' devices and a better knowledge of this type of technology by pathologists will be a fundamental step to continue to develop the use of OCT forensic fields.
\end{abstract}

Keywords OCT in forensic science $\cdot$ Optical coherence tomography $\cdot$ Forensic pathology $\cdot$ Forensic imaging $\cdot$ Forensic entomology $\cdot$ Postmortem ocular changes

\section{Introduction}

Optical coherence tomography (OCT) is an interferometric imaging technique introduced in the mid 1990's which is currently routinely used in some clinical specialties such as ophthalmology. The main principle characterizing OCT relies on the ability of an interferometer to measure the light reflected form a sample of interest to compare it with a reference light, to measure their overlap, or interference.

Figure 1 shows a simple Michelson interferometer. A semireflective mirror (beam-splitter) is struck by light and divided in to two beams. The first will hit a known reflective surface

Matteo Nioi and Pietro Emanuele Napoli contributed equally to this work.

Matteo Nioi

nioimatteo@gmail.com

1 Department of Clinical Sciences and Public Health, Forensic Medicine Unit, University of Cagliari, Cagliari, Italy

2 Department of Surgical Science, Eye Clinic, University of Cagliari, Cagliari, Italy (acting as a 'reference'), the other (the 'signal ray') will impact the tissue being investigated (in this case, the ocular structures). The fixed distances travelled by the beams in the two arms of the interferometer are called "optical paths". The ray reflected by the sample tissue and the one reflected by the reference surface are combined, generating an inference at the semi-reflective mirror. Consequently, when the reflected light exits the interferometer, there will be a signal consisting of the sum of the light reflected by the relevant substrate and the one reflected by the reference surface. Thus, an interference pattern that can be analyzed is obtained when light from both arms have travelled a "similar" optical distance. The intensity of the light emission given by the sum of the two reflected beams can be gathered and measured by a photodetector (spectrometer).

The inference may be constructive or destructive. In the first scenario (Fig. 2), the reflected beams are coherent and in phase (maximum and minimum peaks are coalescent), and the resultant beam is the sum of the two reflected beams. In the other case, the resulting beam width is suppressed, being the maximum and minimum peaks in opposition.

The development of OCT technology has led to a change from devices with mobile mirrors (Time Domain TD-OCT) to 


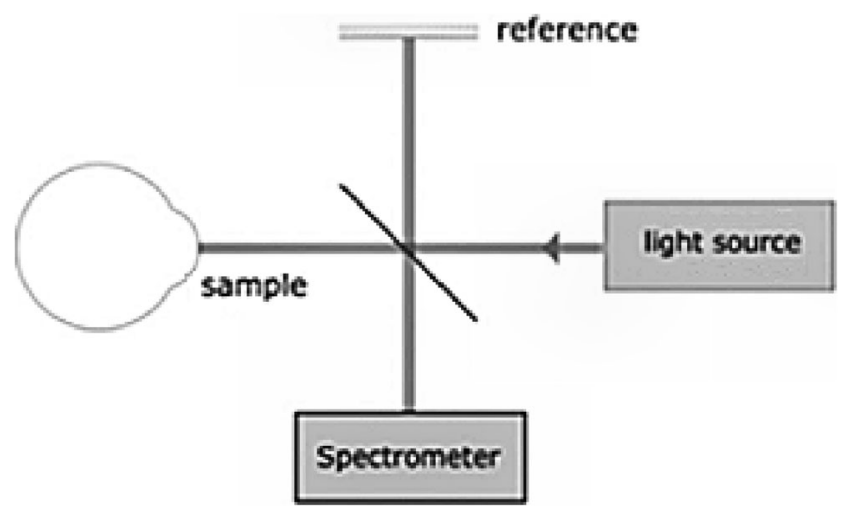

Fig. 1 Diagram of spectral-domain optical coherence tomography (OCT). The light source produces an infrared ray that is divided by a semi-reflected mirror. The two beams are directed towards a reference mirror and the sample being scanned. The two reflective rays are then combined in an 'interference pattern', which is gathered by a detection unit, i.e. the spectrometer

instruments in which mirrors are fixed (Spectral Domain SDOCT, Swept Source-OCT) with significant improvements in image quality and resolution.

Considering the three spatial axes $\mathrm{x}, \mathrm{y}$ and $\mathrm{z}$, two other important OCT features have to be taken into account, namely axial and transversal resolution. Axial resolution is the OCT capability of detecting two points (T; T1) of the sample along the longitudinal direction x. Similarly, for transversal resolution, the two points (A; A1) are situated in the transverse line z. (Fig. 3).

Similar to the techniques used in ultrasound, OCT scanning protocol implies three different modalities - namely, A-scan, B-scan, and C-scan. A-scan, or one-dimensional plot, is referred to the signal reflectivity from a single light beam. Bscans, or two-dimensional scans, are obtained by compiling a number of A-scans from a line of a predetermined length. In this modality, OCT images are quite similar to histological tissue sections, permitting the evaluation of the internal appearance of several structures and the measurement of their thickness. C-scans, or three-dimensional scans, are obtained by compiling B-scans.

Overall, OCT imaging provides several advantages compared with classical histology or autopsy: it allows non-invasive, non-contact, three-dimensional analysis of the tissue being studied in real-time. Although several scholars forecast that advanced imaging techniques may replace the 'classical' autopsy in the very near future [1-6], assuming that 'virtual histology' will be the next step along this path, few studies have focused on the feasibility of OCT application in the forensic field. [7]

In our review, we identified five different topics of potential forensic interest, in which OCT may be a useful diagnostic tool: assessment of the heart and vascularization in the postmortem period, forensic ophthalmology in vivo and after death, clinical forensic medicine, forensic entomology, and different areas of forensic science (the study of blood stains, fingerprints, and hair bulbs for personal identification, as well as the study of materials found in the crime scene for comparation, or anti-fraud investigation).

\section{Material and methods}

We researched four different databases ("PUBMED", "MEDLINE", "SCHOLAR" and "SCIENCE DIRECT") for potential papers from 1995-2019using the following combinations of keywords: "Optical Coherence Tomography" + Forensic, 'Optical Coherence Tomography' + 'postmortem', 'Optical Coherence Tomography + Postmortem + Forensic', 'optical coherence tomography + postmortem + ocular', 'optical coherence tomography after death', and optical coherence tomography+ legal medicine'. From a total of 7339 papers found using these searches, we only selected 57 articles for our review. Specifically, we excluded studies that were purely clinical and were without forensic aims, as well as
Fig. 2 Constructive and destructive interference patterns in OCT imaging. There are two extreme results from the sum of the two returning beams (i.e. the reference and sample arm). A constructive pattern occurs when they are in phase (all positive and negative peaks are perfectly matching, $0^{\circ}$ ). Conversely, a destructive pattern results when the two beams are out of phase $\left(180^{\circ}\right)$
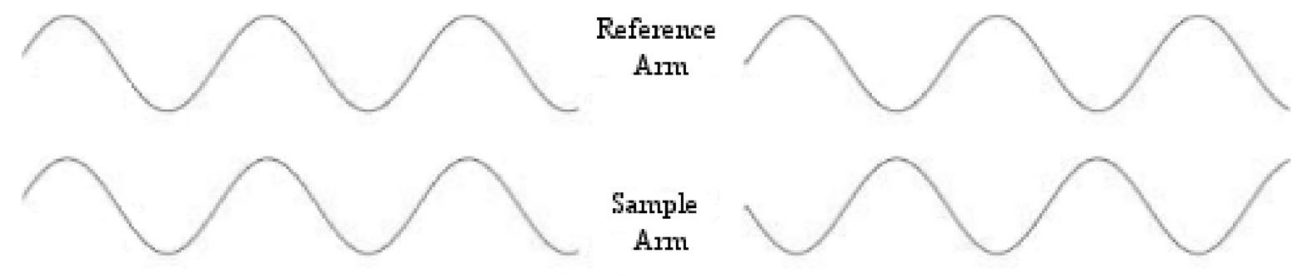

Arm

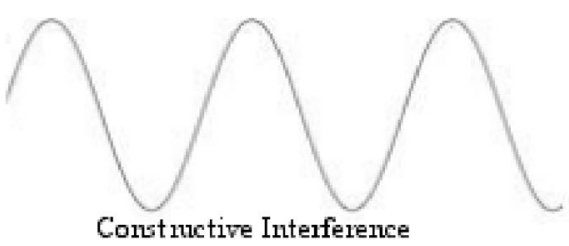

Destuctive Interference 


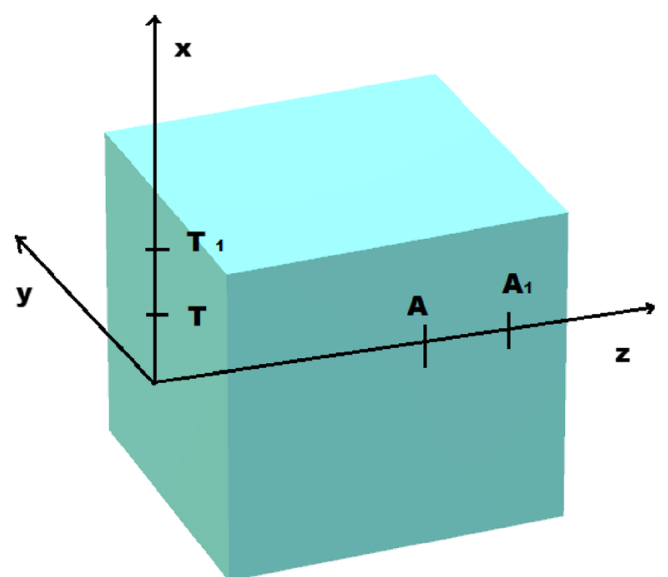

Fig. 3 Reconstruction of the 3D space in OCT imaging. In a Cartesian diagram, the axial resolution represents the OCT capability of detecting two different points $\left(\mathrm{T} ; \mathrm{T}_{1}\right)$ along the $\mathrm{x}$ axis. Conversely, the lateral resolution refers to the OCT ability to disclose two separate points (A; $A_{1}$ ) along the $\mathrm{z}$ axis. The $3 \mathrm{D}$ space is finally constructed by combination of various xz planes

papers with a small or no chance of having potential applications in the forensic field.

\section{Heart and coronary}

The use of imaging techniques as an alternative approach to cadaveric studies has become a reality since 2007 due to the VIRTOPSY project [8]. Despite computed tomography (CT) and magnetic nuclear resonance (MNR) techniques (mainly employed for this purpose) demonstrating their diagnostic role in specific cases (i.e. gunshot wound deaths), there are several situations where a microscopic evaluation of the tissues is mandatory to get a medico-legal diagnosis. One of these cases is when death occurs due to coronary pathology. In 2006, Kume and colleagues presented the first OCT description of different types of coronary thrombin. They studied 108 coronary artery segments in 40 human cadavers, showing that the OCT was not only able to highlight the morphology of the arterial plaques but also to distinguish the red thrombi from the white ones [9]. In 2009, Prati and colleagues hypothesized a clinical application of OCT for the assessment of coronary arteries and in particular its use for atherosclerosis evaluation [10]. In recent work, Adlam et al. compared a single case of post-mortem CT coronary angiography (PMCTA) coupled with OCT to the gross histopathological examination, concluding that the former technique had potential in the diagnosis of cardiac death, being a valid alternative to open autopsy [11]. Recently, some studies have addressed several technical aspects, supporting the need of re-pressurization of vessels for studying the effect of coronary stenosis in vivo and in the postmortem period $[12,13]$. In 2018, Fujii et al. provided a short description of the correlation between the histopathological analysis and OCT images in the visualization of the atherosclerotic plaques, and of the vascular healing response after drug-eluting stent implantation. They concluded that OCT is a promising intracoronary imaging modality for differentiating tissue characteristics (fibrous, calcified, or lipid-rich plaque) and identifying thin-cap fibroatheroma (TCFA) [14].

The results obtained from these various studies strongly suggest a potential role of OCT in the study of coronary artery disease and of sudden cardiac death, even in forensic caseworks as a supplementary diagnostic tool $[15,16]$.

\section{Postmortem ocular findings and clinical forensic ophthalmology}

Ophthalmology is the clinical branch in which the use of OCT has been mainly described. Indeed, the use of this technique is a part of routine practice in various ophthalmologic areas for the study of the retina, the anterior chamber and also the ocular surface [17-20]. Consequently, OCT has also been shown to be useful in cases of forensic interest where ophthalmological diagnosis may be relevant, such as the analysis of retinal signs in cases of shaken baby syndrome [21,22].

Although several studies of forensic pathology have focused on the eye, to date the main area of forensic speculation has dealt with the variation of post mortal biochemistry of the vitreous humor, or at most of the aqueous humor [23-28].

Recently, Napoli and colleagues studied the feasibility of OCT in the detection of postmortem ocular findings, thus detecting a variety of structural changes of a sheep cornea in different phases after death [29-31]. The latter are presumably due to specific and different patho-physiological pathways [32]. A preliminary study in humans allowed detection of novel signs for estimating the postmortem interval (Fig. 4), as the formation of stromal waves in posterior stroma of corneas from the 3rd hour after death (Nioi-Napoli sign). Additional information was also obtained by the postmortem examination of the sclera, irido-corneal angle, vitreous humor, and retina $[33,34]$.

An OCT analysis of inner ocular structures has been proposed by McNabb and colleagues. They were able to retrieve data from the post-mortem retinal observation by means of OCT associated with a periscope. Using the latter, these scholars were able to analyze the nervous tissues, avoiding optical interference of the lens [35].

\section{Particular cases of forensic interest and tissue analysis}

The use of OCT for the objectification of vascular and ophthalmological lesions of forensic interest (i.e. traffic and work- 


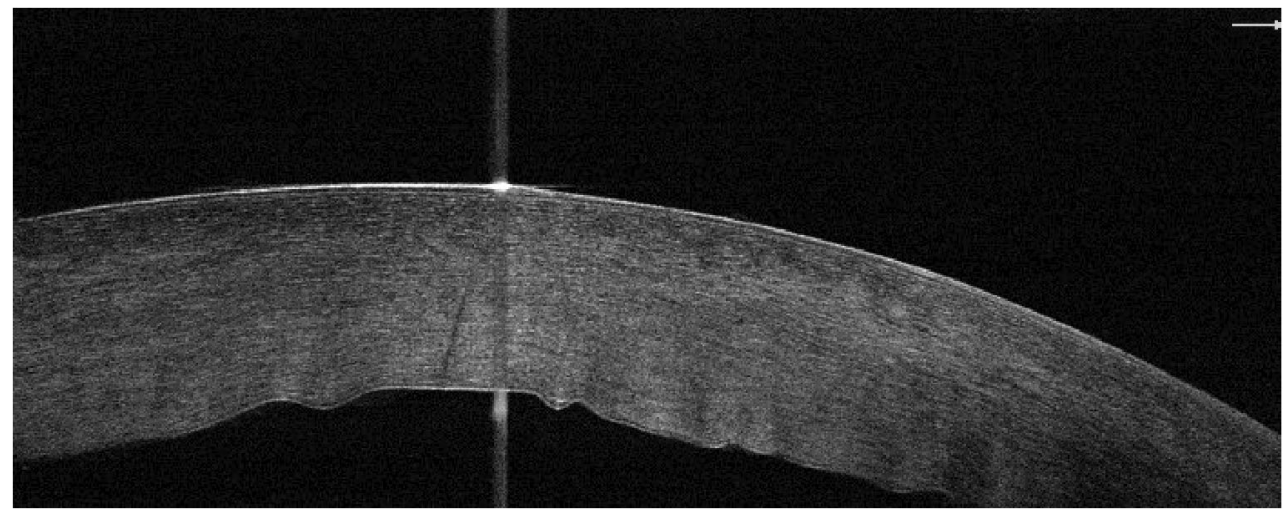

Fig. 4 Morphological appearance of the human cornea after death (NioiNapoli sign). A variable number of morphological changes in corneal tissues are easily detectable in the postmortem period by OCT. Of note, the presence of waves in the posterior stroma are indicative of a time since

related injuries) is already a consolidated reality [36-39]. Some authors have proposed the use of OCT in combination with near-infrared (NIR) vascular imagers in estimating the age of bruise by assessing the degradation of hemoglobin [40]. Again, different preliminary studies have tried to characterize human tissues by means of Spectral Domain-OCT (SD-OCT) alone or in combination with a hyperspectral imaging system (HIS) [41].

\section{Entomology}

The application of OCT in forensic entomology would be to analyze arthropods (including insects, arachnids, centipedes, millipedes) as Calliphora vicina or Chrysomya Rufifacies for post mortem interval (PMI) estimation. In this way, a prolonged, morphological and non-destructive larval study may be achieved [42-46].

\section{Forensic science}

Our literature review revealed the presence of OCT-based studies in several different branches of forensic science.

Choi et al. morphologically analyzed depth-resolved cellular structures of unmodified fresh human scalp hairs with ultrahigh-resolution full-field optical coherence tomography (FF-OCT). The authors supposed that interindividual characteristics could be used in particular cases of personal identification (such as the discrimination of twins by examination of head hairs) [47].

Dubey, Leich, Zhang and Zam applied OCT imaging for the detection of fingerprints. For them, low-coherence interferometry, nonmechanical scanning, and compactness were the main advantages of OCT over conventional fingerprint detection techniques. Other relevant advantages comprised death $\geq 3 \mathrm{~h}$ (Nioi-Napoli sign). As far as we are aware, no other techniques are able to study the human cornea in postmortem intervals in situ, noninvasively, quantitatively, in cross section, and in-depth (histological resolution)

the non-invasive nature of the OCT and the fact that this technique does not require any physical or chemical manipulation of samples [48-51].

Zhang and colleagues studied facial hair follicle distribution by OCT, thus discovering important interindividual differences based on age, gender, and race/ethnicity. They proposed specific patterns of facial hair follicles for each individual that could be useful for forensic identification [52].

In other studies, cross-sectional HD-OCT images were also used to verify the authenticity of banknotes [53, 54] or to characterize vehicle paints $[55,56]$.

Laan and colleagues used OCT for a tridimensional volumetric determination of fresh or dried bloodstains in order to propose a reconstruction of the dynamics of a criminal event [57].

\section{Discussion}

Although OCT imaging has been used with success for several decades in the field of ophthalmology, this technique has encountered meagre employment within the field forensic science, both from practical and research points of view. Nevertheless, characteristics like the lack of physical or chemical sample manipulation, the non-invasiveness, the threedimensionality and the microscopic resolution of the images, are undoubtedly among the most important advantages of this technique and could further encourage its use in different fields of study (i.e. postmortem coronary analysis, postmortem and clinical ophthalmology, forensic entomology, individual identification, forensic sciences purposes).

One of the areas in which application of the OCT method has been demonstrated as effective is the postmortem evaluation of coronary arteries. The autoptic study of coronary arteries is of great importance as it has been estimated that coronary heart disease (CHD) is the leading cause of death in 
adults over the age of 35 in the United States [58]. Although coronary artery disease is generally responsible for clinical signs requiring a complex medical treatment, sudden death may be the first manifestation of it, as coronary modifications are a predisposing factor of arrhythmogenic events [59]. Examination of coronaries using conventional histological techniques presents several limitations, particularly as tissue fixation produces alterations of the arteries [60]. These limitations prompted research of alternative tools for the medicolegal investigation of coronary events. Among these diagnostic approaches, Post-Mortem Computed Tomography Angiography (PMCTA), alone or in combination with OCT, has been widely and successfully applied. Such an approach firstly allows analysis of the tissue without alteration (overcoming the limits of traditional histology) and secondly the achievement of a less invasive approach compared to the classical autopsy techniques, which is also associated with greater accuracy, precision and reliability. There are two possible concerns in using the OCT approach; its higher costs (related to acquisition) compared to traditional techniques (e.g. classical autopsy) and the poor familiarity of pathologists with the new imaging techniques. These problems could be overcome with the marketing of ad hoc tools and adequate preparation of forensic staff.

A more widespread use of OCT techniques may be worthwhile even in investigations in the field of forensic ophthalmology, devoted to the study of post-mortem ocular signs or to the collection of evidence in peculiar clinical ophthalmological cases. Although the appearance of ocular alterations in the postmortem period is a well-known phenomenon that has been widely described in the literature, until now little has been known about their thanatogenesis and they are rarely detected in a systematic way for thanatochrono diagnostic purposes. This is mainly due to the invasiveness of the traditional methods based on histology not only, involving a disfigurement of the body. Failure to carry out an in-depth analysis of the eye during autopsy led to a lack of knowledge of the factors influencing the genesis of post mortal ocular alterations but also a thorough knowledge of the ultrastructural manifestations of the same post mortal phenomena. Although several studies have examined the utility of OCT techniques in the postmortem interval to study the enucleated ocular globes, only recently studies carried out on animal models and human corneas in situ, without enucleation, have demonstrated the feasibility of OCT imaging with excellent results in terms of inter- and intra-individual repeatability. This, in turn, led to the discovery of new signs that cannot be highlighted using traditional methods. An in-depth study in this field can lead not only to a better pathogenic understanding of cadaveric eye signs, but also to the refinement of new methods, based on pachymetry or on morphological alterations or on their combination, useful for estimating the post-mortem interval.
OCT surveys in the field of forensic ophthalmology could prove particularly useful in some selected cases. This approach may guarantee a better probative relevance to the presence, or absence, of retinal modifications in cases of shaken baby syndrome, even in those instances where no other external pathological features are present and retinal findings represent the only sign of abuse. Other fields of application in ophthalmology in living individuals are constituted by the evaluation of the traumatic nature of ocular lesions alleged as a consequence of road accidents or interpersonal violence as well as in cases of chemical burns due to accidental contact with acidic or basic substances occurring at workplace. The knowledge of the main anatomical-clinical ocular alterations related to toxic substances may be relevant in post-mortem analysis. A further area of interest could be the application of OCT in the evaluation of these injuries in postmortem.

Preliminary studies appear to be of great interest for the OCT characterization of human cadaveric tissues. Similarly to what already happens in the characterization of tissues for anatomopathological purposes ex vivo, OCT technology could play a role in the postmortem study of lesions (i.e. wounds of which we should establish the time of onset, study of infant perinatal dura mater and superior sagittal sinus in victims of abusive head trauma) of medical-legal interest.

Other studies carried out on the living have been finalized to the dating of the period of creation of the ecchymoses through the evaluation and degradation of hemoglobin. Although this application of OCT is based on consolidated theoretical bases and is related to a research field (dating of the ecchymoses in the living or differential diagnosis between ecchymosis and hypostasis in the corpse) the practical application of the technique has not found practical confirmation in clinical forensics nor in pathology yet.

The use of OCT could also be of considerable importance in the field of forensic entomology as it would bring significant advantages whenever compared to traditional techniques. The first advantage of this association is the possibility to carry out in vivo measurements. This aspect involves first of all the possibility of not sacrificing the larvae and, as its byproduct, the ability to prospectively study the same individuals in vivo during their developmental stages. This could mean that even a limited number of larvae may be sufficient for an in-depth analysis. The other advantage that could be gained using OCT lies in the OCT's own characteristics, which firstly allow greater precision in measurements and secondly provide the possibility, through tri-dimensional analysis, to study the microscopic characteristics of the sample without destroying it. This would provide new possibilities regarding the examination of new features that might be potentially useful for determining the age of the insect and consequently the postmortem interval.

While on one hand, the use of OCT still appears to be very limited in the field of forensic pathology, forensic medicine 
and entomology, it seems to have been quite successful in the field of forensic sciences.

In this scenario, several studies addressed the determination of identity. A preliminary study has tried, with good results, to examine hair follicles by means of OCT in order to obtain useful information concerning age, sex, and race in particular cases of forensic interest (for example, explosions following which only traces of small cutaneous fragments are available). The results appear encouraging but require further study, and presently constitute a "second choice" with respect to the use of DNA (to be considered as the gold standard). Different groups have attempted to apply OCT to the examination of fingerprints. In these cases the characteristic of OCT that is most important is the possibility of analyzing a substrate in an extremely precise way in a contactless manner and without the use of substances or materials that can potentially alter the test. Even in this case, despite considerable efforts from the experimental point of view, the production of ad hoc tools is not at hand but it could constitute an important step forward for this particular branch of forensic science.

Another field of application is the verification of the authenticity of banknotes. Choi and colleagues, in particular, using the properties of OCT to provide a three-dimensional image and microscopic resolution without modifying the sample, have proposed a method to examine the authenticity of banknotes based on the features of their hologram. This method is undoubtedly comparable to those based on ultraviolet light - spectroscopic or biochemical - but it still requires further refinements for use in the practice of forensic science.

A further field of forensic sciences where the application of OCT technology could be useful is the study of car paints used for criminal purposes - often vehicles are repainted in an attempt to disguise them. Although several methods can currently be used for this purpose (near-infrared spectroscopy, $\mathrm{X}$-ray absorption spectroscopy and micro-spatially offset Raman spectroscopy), they show poor applicability or resolution. Recent studies have shown how the application of OCT, due to the possibility of studying the sample conservatively and the high resolution and precision of the obtainable data, may constitute a promising step forward in this type of evaluation. According to Wang and colleagues, in fact, in a few years the implementation of this techniques to portable instruments will permit a rapid in situ examination of the vehicles, wherever the relevant vehicle is found.

Finally, it is important that an outlook scan be obtained in the study of blood stains. This technique is indeed an excellent tool for investigating the crystallization of the sample and its three-dimensional evaluation. The results could be of extreme interest if experiments acquiring morphological data were combined with data concerning hemoglobin degradation, useful for determining the time the blood spot was deposited.

In conclusion, although OCT devices provide valuable technical features such as the possibility of being used without causing alterations of the examined substrate (that can be studied in a perspective manner) and the capability of obtaining three-dimensional data with a resolution comparable to histological ones, this technique is still scarcely employed in the forensic field. Considering the possible advantages of using OCT, it appears easily foreseeable that development of experimental studies that can fully exploit all the main characteristics of this technique will lead to considerable progress in different areas of forensic science.

\section{Key points}

1. OCT (optical coherence tomography) is based on an optical measurement technique using low frequency interferometry. Similar to echotomography, OCT permits the study of a tissue or a surface through the analysis of data obtained from the reflection of a laser beam. The advantages given by its application are non-invasiveness (absence of contact), three-dimensionality and the high resolution of data, which can be interpreted in real time.

2. The technique has been used very successfully in ophthalmology: it was introduced in 1991 and is currently widely used by clinicians. On the contrary, despite indisputable advantages, it is rarely used in the forensic sciences, both in practice and in research.

3. The use of OCT in the postmortem period may lead to important advances in forensic pathology for the study of cardiac, vascular and ocular tissues.

4. In forensic entomology, the use of OCT may ameliorate the study of insects in a perspective manner without the need for their sacrifice.

5. OCT may have important repercussions in forensic sciences since it may be useful for verifying documents, identifying of traces of paint, studying fingerprints, and describing the ultrastructural characteristics of blood stains.

\section{References}

1. Roberts IS, Benamore RE, Benbow EW, Lee SH, Harris JN, Jackson A, et al. Post-mortem imaging as an alternative to autopsy in the diagnosis of adult deaths: a validation study. Lancet. 2012;379:136-42.

2. Thali MJ, Yen K, Schweitzer W, Vock P, Boesch C, Ozdoba C, et al. Virtopsy, a new imaging horizon in forensic pathology: virtual autopsy by postmortem multislice computed tomography (MSCT) and magnetic resonance imaging (MRI)-a feasibility study. J Forensic Sci. 2003;48:386-403.

3. Bolliger SA, Thali MJ, Ross S, Buck U, Naether S, Vock P. Virtual autopsy using imaging: bridging radiologic and forensic sciences. A review of the Virtopsy and similar projects. Eur Radiol. 2008;18: $273-82$. 
4. Rutty GN, Morgan B. Future evidence in forensic imaging. In: Ferrara SD, editor. P5 medicine and justice. Boston: Springer; 2017. p. $576-85$.

5. Rutty GN, Morgan B, O’Donnell C, Leth PM, Thali MJ. Forensic institutes across the world place CT or MRI scanners or both into their mortuaries. J Trauma. 2008;65:493-4.

6. Morgan B, Rutty GN. How does post-mortem imaging compare to autopsy, is this a relevant question? J Forensic Radiol Imaging. 2016;4:2-6.

7. Thali MJ, Dirnhofer R, Becker R, Oliver W, Potter K. Is 'virtual histology' the next step after the 'virtual autopsy'? Magnetic resonance microscopy in forensic medicine. Magn Reson Imaging. 2004:22:1131-8.

8. Thali MJ, Jackowski C, Oesterhelweg L, Ross SG, Dirnhofer R. VIRTOPSY-the Swiss virtual autopsy approach. Legal Med. 2007;9:100-4.

9. Kume T, Akasaka T, Kawamoto T, Ogasawara Y, Watanabe N, Toyota E, et al. Assessment of coronary arterial thrombus by optical coherence tomography. Am J Cardiol. 2006;97:1713-7.

10. Prati F, Regar E, Mintz GS, Arbustini E, Di Mario C, Jang IK, et al. Expert review document on methodology, terminology, and clinical applications of optical coherence tomography: physical principles, methodology of image acquisition, and clinical application for assessment of coronary arteries and atherosclerosis. Eur Heart J. 2009;31:401-15.

11. Adlam D, Joseph S, Robinson C, Rousseau C, Barber J, Biggs M, et al. Coronary optical coherence tomography: minimally invasive virtual histology as part of targeted post-mortem computed tomography angiography. Int J Legal Med. 2013;127:991-6.

12. Adnan A, Robinson C, Biggs M, Joseph S, Morgan B, Rutty GN, et al. Optical coherence tomography of re-pressurised porcine coronary arteries: a systematic study. J Forensic Radiol Imaging. 2016;4:53-7.

13. Robinson C, Adnan A, Adlam D, Biggs M, Rutty GN, Morgan B. Measuring pressure during coronary artery angiography in ex-vivo hearts. J Forensic Radiol Imaging. 2016;4:58-62.

14. Fujii K, Kawakami R, Hirota S. Histopathological validation of optical coherence tomography findings of the coronary arteries. J Cardiol. 2018;72:179-85.

15. Michaud K, Grabherr S, Jackowski C, Bollmann MD, Doenz F, Mangin P. Postmortem imaging of sudden cardiac death. Int $\mathrm{J}$ Legal Med. 2014;128:127-37.

16. Morgan B, Adlam D, Robinson C, Pakkal M, Rutty GN. Adult post-mortem imaging in traumatic and cardiorespiratory death and its relation to clinical radiological imaging. Brit J Radiol. 2014;87: 20130662.

17. Napoli PE, Nioi M, d'Aloja E, Fossarello M. The Bull's eye pattern of the tear film in humans during visual fixation on en-face optical coherence tomography. Sci Rep. 2019;9:1413.

18. Napoli PE, Coronella F, Satta GM, Galantuomo MS, Fossarello M. Evaluation of the adhesive properties of the cornea by means of optical coherence tomography in patients with meibomian gland dysfunction and lacrimal tear deficiency. PLoS One. 2014;9: e115762.

19. Galantuomo MS, Fossarello M, Cuccu A, Farci R, Preising MN, Lorenz B, et al. Rebound macular edema following oral acetazolamide therapy for juvenile X-linked retinoschisis in an Italian family. Clin Ophthal. 2016;10:2377-82.

20. Napoli PE, Cuccu A, Farci R, Fossarello M. Simultaneous occlusion of three cilioretinal arteries following scleral buckling surgery under local anesthesia. Int Med Case Rep J. 2016;9:285-90.

21. Arredondo JL, Fernandes JR, Rao C. Ocular findings in pediatric deaths under 2 years of age (1994-2004). J Forensic Sci. 2008;53: 928-34.
22. Muni RH, Kohly RP, Sohn EH, Lee TC. Hand-held spectral domain optical coherence tomography finding in shaken baby syndrome. Retina. 2010;30:S45-50.

23. Madea B, Rödig A. Time of death dependent criteria in vitreous humor-accuracy of estimating the time since death. Forensic Sci Int. 2006; 164:87-92.

24. Henssge C, Madea B. Estimation of the time since death. Forensic Sci Int. 2007;165:182-4.

25. Gottardo R, Palacio C, Shestakova KM, Moskaleva NE, Bortolotti F, Tagliaro F. A new method for the determination of ammonium in the vitreous humour based on capillary electrophoresis and its preliminary application in thanatochemistry. CCLM. 2019;57:504-9.

26. Locci E, Scano P, Rosa MF, Nioi M, Noto A, Atzori L, et al. A metabolomic approach to animal vitreous humor topographical composition: a pilot study. PLoS One. 2014;9:e97773.

27. Rosa MF, Scano P, Noto A, Nioi M, Sanna R, Paribello F, et al. Monitoring the modifications of the vitreous humor metabolite profile after death: an animal model. Biomed Res Int. 2015;2015: 627201.

28. Zelentsova EA, Yanshole LV, Snytnikova OA, Yanshole VV, Tsentalovich YP, Sagdeev RZ. Post-mortem changes in the metabolomic compositions of rabbit blood, aqueous and vitreous humors. Metabolomics. 2016;12:172.

29. Napoli PE, Nioi M, d'Aloja E, Fossarello M. Post-mortem corneal thickness measurements with a portable optical coherence tomography system: a reliability study. Sci Rep. 2016;6:30428.

30. Iovino C, Napoli PE, Nioi M, Sanna R, Paribello F, d'Aloja E, et al. Intraobserver variability of post-mortem corneal thickness measurements by using a portable OCT system. Invest Ophthalmol Vis Sci. 2017;58:3516. https://iovs.arvojournals.org/article.aspx?articleid= 2640805\&resultClick=1. Accessed 14 July 2019

31. Nioi M, Napoli PE, Iovino C, Paribello F, Fossarello M, d'Aloja E. Corneal findings after death: a preliminary OCT study on an animal model. Invest Ophthalmol Vis Sci. 2017;58:4873.https://iovs. arvojournals.org/article.aspx ?articleid=2638196\&resultClick=1 . Accessed 14 July 2019

32. Nioi M, Napoli PE, Demontis R, Locci E, Fossarello M, d'Aloja E. Morphological analysis of corneal findings modifications after death: a preliminary OCT study on an animal model. Exp Eye Res. 2018;169:20-7.

33. Nioi M, Napoli PE, Paribello F, Demontis R, De-Giorgio F, Porru E, et al. Use of optical coherence tomography on detection of postmortem ocular findings: pilot data from two cases. J Int OMICS. 2018. https://doi.org/10.5584/jiomics.v8i1.226.

34. Linnola RJ, Findl O, Hermann B, Sattmann H, Unterhuber A, Happonen RP, et al. Intraocular lens-capsular bag imaging with ultrahigh-resolution optical coherence tomography: Pseudophakic human autopsy eyes. J Cataract Refract Surg. 2005;31:818-23.

35. McNabb RP, Tian J, Farsiu S, Izatt JA, Lad EM, Kuo AN. Retinal imaging in human autopsy eyes using a custom optical coherence tomography periscope. Biomed Optics Exp. 2017;8:415-9.

36. Napoli PE, Nioi M, Iovino C, Sanna R, d'Aloja E, Fossarello M. Ocular surface and respiratory tract damages from occupational, sub-chronic exposure to fluorspar: case report and other considerations. Int Ophthalmol. 2019;39:1175-8.

37. Napoli PE, Sanna R, Iovino C, Fossarello M. Resolution of pinguecula-related dry eye disease after argon laser photocoagulation. Int Med Case Rep J. 2017;10:247-50.

38. Cheshire EC, Malcomson RD, Joseph S, Adnan A, Adlam D, Rutty GN. Post-mortem imaging of the infant and perinatal dura mater and superior sagittal sinus using optical coherence tomography. Int J Legal Med. 2017;131:1377-83.

39. Kivlin JD, Currie ML, Greenbaum VJ, Simons KB, Jentzen J. Retinal hemorrhages in children following fatal motor vehicle crashes: a case series. Arch Ophthalmol. 2008;126:800-4. 
40. Kim O, Lines C, Duffy S, Albert M, Crawford G. Modeling and measuring extravascular hemoglobin: aging contusion. In: Schlesinger M, editor. Applications of electrochemistry in medicine. Boston: Springer; 2013. p. 381-401.

41. Dontu S, Miclos S, Savastru D, Tautan M. Combined spectraldomain optical coherence tomography and hyperspectral imaging applied for tissue analysis: preliminary results. App Surface Sci. 2017;417:119-23.

42. Ma T, Huang J, Wang JF. Study on the pupal morphogenesis of Chrysomyarufifacies (Macquart)(Diptera: Calliphoridae) for postmortem interval estimation. Forensic Sci Int. 2015;253:88-93.

43. Voss SC, Magni P, Dadour I, Nansen C. Reflectance-based determination of age and species of blowfly puparia. Int J Legal Med. 2017;131:263-74.

44. Choi KS, Wijesinghe RE, Lee C, Lee SY, Jung HY, Jeon M, et al. In vivo observation of metamorphosis of Plodia interpunctella Hübner using three-dimensional optical coherence tomography. Ent Res. 2017;47:256-62.

45. Ravichandran NK, Wijesinghe RE, Lee SY, Choi KS, Jeon M, Jung HY. Non-destructive analysis of the internal anatomical structures of mosquito specimens using optical coherence tomography. Sensors. 2017;17:1897.

46. Brown K, Harvey M. Optical coherence tomography: age estimation of Calliphoravicina pupae in vivo? Forensic Sci Int. 2014;242: 157-61.

47. Choi WJ, Min G, Lee BH, Pi LQ. Lee WS qualitative investigation of fresh human scalp hair with full-field optical coherence tomography. J Biomed Opt. 2012;17:036010.

48. Dubey SK, Anna T, Shakher C, Mehta DS. Fingerprint detection using full-field swept-source optical coherence tomography. Appl Phys Lett. 2007;91:181106.

49. Leich M, Kiltz S, Dittmann J, Vielhauer C. Non-destructive forensic latent fingerprint acquisition with chromatic white light sensors. Proc SPIE 7880, Media Watermarking, Security, and Forensics III 2011;https://doi.org/10.1117/12.872331.

50. Zhang N, Wang C, Sun Z, Li Z, Xie L, Yan Y, et al. Detection of latent fingerprint hidden beneath adhesive tape by optical coherence tomography. Forensic Sci Int. 2018;287:81-7.

51. Zam A, Dsouza R, Subhash HM, O'Connell ML, Enfield J, Larin K, et al. Feasibility of correlation mapping optical coherence tomography (cmOCT) for anti-spoof sub-surface fingerprinting. $\mathrm{J}$ Biophotonics. 2013;6:663-7.
52. Zhang N, Wang C, Li Z, Xie L, Guo J, Xu L, et al. Preliminary analysis of facial hair follicle distribution for forensic identification using OCT. Proc SPIE 10497, imaging, manipulation, and analysis of biomolecules, Cells, and Tissues XVI. 2018; https://doi.org/10. $1117 / 12.2285309$.

53. Choi WJ, Min GH, Lee BH, Eom JH, Kim JW. Counterfeit detection using characterization of safety feature on banknote with fullfield optical coherence tomography. J Optical Soc Korea. 2010;14: 316-20.

54. Takalo J, Timonen J, Sampo J, Rantala M, Siltanen S, Lassas M. Using the fibre structure of paper to determine authenticity of the documents: analysis of transmitted light images of stamps and banknotes. Forensic Sci Int. 2014;244:252-8.

55. Zhang N, Wang $\mathrm{C}$, Sun $\mathrm{Z}$, Mei H, Huang W, Xu L, et al. Characterization of automotive paint by optical coherence tomography. Forensic Sci Int. 2016;266:239-44.

56. Wang C, Zhang N, Sun Z, Li Z, Li Z, Xu X. Recovering hidden sublayers of repainted automotive paint by $3 \mathrm{D}$ optical coherence tomography. Aust J Forensic Sci. 2017;51:331-9.

57. Laan N, Bremmer RH, Aalders MC, de Bruin KG. Volume determination of fresh and dried bloodstains by means of optical coherence tomography. J Forensic Sci. 2014;59:34-41.58.

58. Sanchis-Gomar F, Perez-Quilis C, Leischik R, Lucia A. Epidemiology of coronary heart disease and acute coronary syndrome. Ann Transl Med. 2016;4:256.

59. Chatterjee NA, Moorthy MV, Pester J, Schaecter A, Panicker GK, Narula D. Sudden death in patients with coronary heart disease without severe systolic dysfunction. JAMA Cardiol. 2018;3:591600 .

60. Siegel RJ, Swan K, Edwalds G, Fishbein MC. Limitations of postmortem assessment of human coronary artery size and luminal narrowing: differential effects of tissue fixation and processing on vessels with different degrees of atherosclerosis. J Am Coll Cardiol. 1985;5:342-6.

Publisher's note Springer Nature remains neutral with regard to jurisdictional claims in published maps and institutional affiliations. 